\title{
Um modelo de organização e partilha de informação de enfermagem entre hospital e centro de saúde: estudo delphi
}

\author{
A model of organization and nursing exchange information: a delphi study \\ Un modelo de organización e intercambio de información de enfermeria entre \\ un hospital y un centro de salud: estudio delphi
}

\author{
Paulino Artur Ferreira de Sousa', Marta Hansen Lima Basto Correia Frade ${ }^{2}$, \\ Denisa Maria de Melo Vasques de Mendonça ${ }^{3}$
}

\begin{abstract}
RESUMO
Introdução: Uma partilha adequada de informação de enfermagem deve ser encarada como estratégia fundamental para a melhoria da continuidade de cuidados. A informação disponível e o encaminhamento da mesma na Unidade Local de Saúde de Matosinhos - Portugal, não tem dado uma resposta adequada às necessidades de informação sentidas pelos enfermeiros.Objetivos: Este artigo apresenta o desenvolvimento e a definição de um modelo de organização e partilha de informação de enfermagem entre Hospital e Centro de Saúde.Métodos: O estudo foi desenvolvido recorrendo à Técnica Delphi, com três rounds, integrando um painel de enfermeiros em exercício de funções nas diferentes áreas de actuação. Resultados: Os consensos obtidos permitiram chegar a uma hierarquização de aspectos centrais do problema em estudo, nomeadamente dos pressupostos que devem guiar o desenvolvimento do sistema e da informação que deverá efectivamente ser partilhada entre hospital e centro de saúde, com indicação de níveis de prioridade de acesso.Conclusões: A articulação entre os diferentes contextos de cuidados de saúde deverá ser assegurada por um modelo que garanta a partilha recíproca e confidencial da informação de enfermagem, em tempo real, com recurso às Tecnologias de Informação e Comunicação.
\end{abstract}

Descritores: Enfermagem; Sistemas de informação; Registros de enfermagem

\begin{abstract}
Background: An adequate exchange of nursing information between health care facilities should be taken as a fundamental strategy to promote continuity of nursing care. The actual system used to exchange nursing information between nursing staff from health care services in Matosinhos, Portugal, appears to be unsatisfactory. Objective: This paper reports the development and use o a model of organization and nursing information exchange. Methods: The study used the Delphi technique whose focus was to reach a consensus from a panel of expert nurses from different areas of practice. Results: The consensus of the panel allowed reaching hierarchical aspects of the problem in exchanging nursing information. It was possible to define the presupposed information that should guide the Nursing Exchange Information System development, and to identify the information that effectively should be exchanged between health care facilities at different levels of access. Conclusion: The exchange of information among different health care facilities should be guided by a model that guarantees the reciprocal and confidential nursing information, in real time, using appropriate information and communication technologies.
\end{abstract}

Keywords: Nursing; Information systems; Nursing records.

\section{RESUMEN}

Encuadramiento: Lo comparto de información de enfermería debe enfrentarse como una estrategia fundamental para la mejora de la continuidad de cuidados. La información disponible y lo encaminamiento de la misma en la Unidad Local de Salud de Matosinhos, no han estado dando una respuesta correcta a las necesidades de la información de los enfermeros. Objetivo: Este artiículo informa el desarrollo y la definición de un modelo de organización y comparto de información de enfermería entre hospital e centros de salud.Método: El estudio que se retiró en una técnica de Delphi se desarrolló, con tres rondas que integran un painel de enfermeros de diferentes áreas de actuación.Resultados: Los consensos obtenidos permitieron la jerarquizacíon de los aspectos principales de lo problema en estudio. Son presentados los consensos relativamente a los presupuestos que deben guiar el desarrollo del Sistema de Comparto de Información de Enfermería y la información que deberá efectivamente ser compartida entre hospital y centro de salud, con indicación de los niveles de prioridad de acceso.Conclusión: La articulación entre los diferentes medios de salud debe ser asegurada por un modelo que garantiza lo comparto recíproco y confidencial de la información de enfermería, en tiempo real, con recurso a las tecnologías de la información y comunicación.

Descriptores: Enfermería; Sistemas de información; Registros de enfermería

1 Professor Coordenador da Escola Superior de Enfermagem de São João, Unidade de Investigação: Sistemas de Informação em Enfermagem - USINE - Porto-Portugal.

2 Coordenadora Científica da Unidade de Investigação e Desenvolvimento em Enfermagem - Lisboa-Portugal.

3 Professora Associada do Instituto de Ciências Biomédicas de Abel Salazar, Departamento de Estudos Populações, Laboratório de Biometria - Porto-Portugal. 


\section{INTRODUÇÃO}

No contexto das sociedades actuais a informação assume uma grande importância no âmbito da saúde. Por isso, não é de estranhar uma preocupação crescente com o desenvolvimento de Sistemas de Informação (SI) eficientes que permitam a maximização da gestão dos serviços e promovam a melhoria da qualidade dos cuidados de saúde. As dificuldades sentidas na gestão da informação na saúde têm levado governos e entidades responsáveis, bem como profissionais de saúde, a demonstrar um crescente envolvimento nos processos de desenvolvimento de SI, que permitam uma utilização racional e eficiente da informação, tendo em vista a melhoria da qualidade dos cuidados. É consensual a necessidade básica de aceder à informação gerada pelos enfermeiros, garantindo que esse acesso se torne extensivo a todos os profissionais de saúde, de forma rápida e eficaz. Mas, para que se possa promover a sua partilha nas diferentes áreas, torna-se necessário que se proceda à gestão e organização deste recurso, definindo com clareza que tipo de informação deverá ser documentada, e desta, qual deverá ser partilhada, de forma a consolidar um Sistema de Informação de Enfermagem (SIE) adequado.

No sector da saúde, é fundamental que os SI assegurem a informação útil e necessária às diversas funções dos profissionais de saúde e aos diferentes níveis da instituição. Para isso, é essencial definir SI, que constituam estruturas sólidas, capazes de reunir, guardar, processar e facultar informação relevante, de modo a torná-la acessível e útil para aqueles que a desejam (e possam) utilizar. É neste sentido que optimizar o fluxo de informação numa instituição de saúde precisa de ser encarado como estratégia fundamental para a melhoria da qualidade de cuidados prestados ao cidadão.

As principais limitações para o desenvolvimento de qualquer SIE incluem a pouca familiaridade dos enfermeiros com a tecnologia, a sua escassa participação no processo de decisão na aquisição de equipamentos, o não envolvimento efectivo no desenvolvimento dos sistemas, o elevado custo da implementação das tecnologias, as diferenças entre conhecimento formal e informal, a falta de estruturação dos dados, a inexistência de um conjunto mínimo de dados e o acesso parcial por parte dos enfermeiros a estes dados ${ }^{(1)}$. Complementando esta ideia, Marin ${ }^{(2)}$ acrescenta a necessidade urgente de desenvolvimento de uma base de dados de saúde a nível nacional e de uniformização das classificações ou vocabulários de enfermagem, por forma a optimizar a comunicação, tendo em vista a falta de homogeneidade nas várias iniciativas actualmente existentes para solucionarem o problema ${ }^{(2-3)}$.

Quando nos centramos na gestão dos SIE na organização, nomeadamente na articulação entre os diferen- tes contextos de cuidados de saúde, verificamos que os actuais SIE reflectem uma estrutura dos serviços de saúde dividida entre "Centros de Saúde" e "Hospitais". A troca de informação entre os serviços de saúde é escassa ou inexistente. $\mathrm{O}$ acesso à informação não é extensivo a todos os profissionais de saúde, de forma rápida e eficaz. A deficiente articulação/circulação de informação entre estes contextos de cuidados de saúde, relacionada com a forma como a informação é disponibilizada aos seus utilizadores, não permite uma resposta adequada às necessidades de informação sentidas pelos enfermeiros, dificultando a tomada de decisão em enfermagem para a continuidade de cuidados entre os diferentes contextos das práticas.

A falta de informação e encaminhamento da mesma dentro do sistema, associada à ausência de uma estratégia de integração de recursos e de articulação de contextos de cuidados, agrava a dificuldade de acesso aos meios existentes em tempo útil. Por isso, a necessidade de ultrapassar a dicotomia "Centro de Saúde / Hospital", através do desenvolvimento de Sistemas Locais de Saúde efectivamente articulados como uma rede de referência hospitalar, implica o estabelecimento de linhas de articulação onde os SIE são preponderantes.

Davenport alerta-nos para o fato de muitas vezes, o fascínio das tecnologias fazer esquecer o verdadeiro sentido da informação, que é informar. Por outro lado, salienta que de nada servirá a existência de "todos os computadores do mundo", se os utilizadores não estiverem interessados na informação que eles podem gerar $^{(4)}$. É neste sentido que, muitas vezes, grande parte dos dados partilhados entre os profissionais não se transformam em informação, por falta de integridade referencial, por duplicação, por pouca confiabilidade e credibilidade, por falta de interactividade entre os diversos SI. Por isso, inúmeras vezes, as decisões face às situações que requerem cuidados de enfermagem são tomadas sem as informações necessárias. As barreiras ao fluxo e partilha de informação são imensas, fazendo com que as informações que agregariam valor cheguem tarde de mais. Isto porque a informação que é relevante num determinado momento, pode deixar de o ser depois. Torna-se necessário estabelecer ou reforçar novas relações entre os diversos intervenientes dos Sistemas de Saúde, permitindo o estabelecimento de novos canais com os utilizadores, disponibilizando-lhes mais informação( ${ }^{(4)}$.

Quando pensámos no desenvolvimento de um Sistema de Partilha de Informação de Enfermagem (SPIE) entre os diferentes contextos de cuidados de saúde (Hospital e Centros de Saúde), emergiram algumas questões, nomeadamente:

- Que informação processada pelos enfermeiros nos diferentes contextos de cuidados de saúde deverá ser partilhada para assegurar a continuidade de cuidados de enfermagem? 
- Que estratégias de partilha de informação de enfermagem deverão existir entre os diferentes contextos de cuidados de saúde para assegurar a sua interligação com fiabilidade, eficiência e segurança?

- Que modelo de organização e partilha de informação de enfermagem deverá existir entre os diferentes contextos de cuidados de saúde?

Este estudo foi realizado entre 2001-2004, no contexto de um estudo mais alargado centrado numa abordagem da "informação relevante para efeitos da continuidade nos cuidados de enfermagem entre diferentes contextos de cuidados (Hospital e Centro de Saúde)", que emerge de um percurso de investigação-ação, desenvolvido na Unidade Local de Saúde de Matosinhos (Portugal). Esta unidade encontra-se integrada no Serviço Nacional de Saúde, representando um novo modelo de organização dos serviços prestadores de cuidados de saúde primários e hospitalares.

O sistema de saúde português é constituído por diferentes sectores que se complementam. Um desses sectores corresponde ao conjunto de instituições e serviços, dependentes do Ministério da Saúde, que têm como missão garantir o acesso de todos os cidadãos aos cuidados de saúde, que se encontram organizados num Serviço Nacional de Saúde. De acordo com a Lei de Bases da Saúde, o sistema de saúde assenta nos cuidados de saúde primários, que devem situar-se junto das comunidades, devendo ser promovida a intensa articulação entre os cuidados de saúde, reservando a intervenção dos mais diferenciados para as situações deles carecidas e garantindo a circulação recíproca e confidencial da informação clínica relevante sobre os utentes ${ }^{(5)}$.

\section{MÉTODOS}

O recurso a grupos para gerar ideias, consensos e tomar decisões sobre a partilha de informação de enfermagem parece ser uma forma adequada para procurar algumas respostas. O consenso é

o resultado de um processo de interacção grupal, presencial ou anônimo, em que os participantes têm a oportunidade de trocar informações entre si sobre o assunto em estudo até atingirem o nível de acordo previamente fixado ou estabelecido pelo grupo ${ }^{(6)}$.

A técnica de Delphi foi escolhida para gerar consensos entre os enfermeiros por forma a responder às questões apresentadas.

\section{Técnica delphi}

O recurso à técnica Delphi permite estruturar o processo de comunicação de um grupo de indivíduos, como um todo, de forma a permitir que partilhem o debate de um assunto complexo, tendo como fonte de informação a opinião de peritos. O seu objetivo é a obtenção de uma opinião coletiva qualificada sobre determinadas questões, a partir de um grupo de pessoas seleccionadas. Tal abordagem é indicada para situações onde não existem informações precisas ou dados históricos, ou em situações onde se deseja estimular novas ideias ${ }^{(6-10)}$.

As ideias que emergem têm em conta os conhecimentos, a experiência e a visão estratégica de julgar e predizer mudanças a longo prazo dos peritos, no sentido de "prever o futuro" e/ou intervir na sua configuração ${ }^{(10)}$. Partindo das opiniões, pontos de acordo e divergências entre os peritos, pretende-se chegar a certos elementos de consenso e a uma hierarquização de aspectos importantes e secundários relativos ao problema em estudo. Diferente de outras estratégias de investigação, o seu objetivo não é deduzir uma simples resposta ou chegar unicamente ao consenso, mas obter respostas e opiniões de qualidade para uma dada questão apresentada ao painel de especialistas ${ }^{(11)}$.

As principais características da técnica Delphi consistem na utilização de um painel de peritos para obter consenso, sustentado no facto de os participantes não terem confrontação frente a frente, na garantia de anonimato das respostas e no uso de ferramentas estatísticas simples para identificar níveis de concordância. Por isso, associam-se a esta técnica as seguintes características básicas:

- Anonimato: a opinião dos membros do grupo é obtida através de um questionário, pelo que os participantes não interagem directamente com os outros;

- Feedback: os resultados são enviados aos participantes para que estes validem novamente as suas opiniões. O objectivo básico do feedback é distribuir todas as informações disponíveis e geradas pelo grupo entre os seus participantes;

- Interacção: a técnica consiste em sucessivos rounds cuja quantidade é determinada de acordo com um critério de consenso do grupo ou estabilização dos julgamentos individuais;

- Resposta estatística do grupo: a opinião do grupo é definida, no final de cada round, através da agregação das opiniões individuais ${ }^{(7,12-13)}$.

Na prática, este "feedback" controlado consiste em remeter a todos os participantes, em rounds sucessivos, a informação que se vai gerando em cada etapa ${ }^{(14)}$. É neste sentido que o investigador, recorrendo a esta estratégia, vai transmitindo aos participantes a informação livre de interferências, eliminando aquela que considera não relevante, repetida e confusa, com o objectivo de enviar uma mensagem clara (tanto em conteúdo, como na forma e linguagem), que agrupe a opinião do grupo 
sobre a área em discussão ${ }^{(15)}$. Desta forma, comunicando aos participantes um resumo da etapa precedente, reduz o "ruído semântico", fornecendo ao grupo somente aquilo que se refere aos objectivos e metas do estudo, evitando que os participantes se desviem dos pontos centrais do problema.

\section{Selecção dos membros do painel}

Foram duas as preocupações que orientaram a selecção dos participantes do painel: o número de participantes e a escolha dos informadores-chave. Não existe a exigência de um número mínimo ou máximo de componentes do painel, podendo variar de um pequeno grupo até um grupo numeroso, dependendo do tipo de problema a ser investigado e da população e/ou amostra utilizáveis $^{(13)}$.

A decisão pela constituição deste painel resultou da necessidade de obtenção de opiniões, onde a diversidade era uma garantia de pontos de vista das diferentes áreas de exercício profissional de enfermagem. A seleção da amostra para integrar o painel de peritos deverá ser considerada como não aleatória de conveniência ou intencional. O painel foi constituído por enfermeiros, em exercício de funções nas diferentes áreas de enfermagem (prestação de cuidados, gestão de cuidados e ensino de enfermagem), uns que considerámos "peritos" e outros "afectados"(16). Foram considerados "peritos", os enfermeiros que possuíam conhecimentos, experiência, capacidade predictiva e objectividade, tal como é entendido o conceito clássico de perito. Os "peritos" na área em estudo foram selecionados com base no seu conhe- cimento sobre a respectiva área e envolvimento no estudo em questão, isto é, por possuírem informações e experiências relevantes para o processo ${ }^{(14,17)}$. Os "afetados" não se distinguem por possuir mais ou menos conhecimentos relevantes na área do objeto de estudo, mas por se encontrarem implicados, de alguma maneira, no processo de criação do sistema.

A abordagem ao grupo de peritos foi concretizado através de questionários. O objetivo dos questionários utilizados para concretizar os diferentes rounds determinou o recurso a diferentes constituições do painel de participantes, fato que será explicitado na descrição de cada um destes.

\section{Critérios de consenso}

As respostas individuais às questões apresentadas nos diferentes questionários, permitiram uma "resposta estatística de grupo" que garantiu um resultado final do grupo de participantes para análise sobre consenso obtido. Por se tratar de um estudo de consenso, utilizaramse os recursos da estatística descritiva, mais especificamente as medidas de tendência central (média, moda e mediana) e de dispersão (desvio-padrão, coeficiente de variação e amplitude inter-quartil), sendo analisadas, também, as frequências dos scores ${ }^{(18-21)}$.

$\mathrm{Na}$ definição do tipo de consenso, tivemos em consideração a análise dos scores obtidos em cada uma das questões, pelo que o consenso foi atingindo sempre que, relativamente a cada item, se verificaram as condições apresentadas nas Tabelas 1 .

Tabela 1 - Tipo de Consenso (adaptado de GOOSSEN ${ }^{(19)}$ )

\begin{tabular}{|c|c|}
\hline Consenso & Definição a) \\
\hline $\begin{array}{l}\text { Consenso (critério de } \\
\text { inclusão) }\end{array}$ & $\begin{array}{l}\text { Mediana - igual ou superior a } 6 \text {; } \\
\text { Mais de } 75 \% \text { dos membros do painel de peritos encontram-se entre os scores } 5 \text { e } 7 \text {; } \\
\text { Ausência de comentários de membros do painel de peritos que indiquem ambiguidades ou má } \\
\text { compreensão das questões. }\end{array}$ \\
\hline $\begin{array}{l}\text { Consenso (critério de } \\
\text { exclusão) }\end{array}$ & $\begin{array}{l}\text { Mediana - igual ou inferior a } 2 \text {; } \\
\text { Mais de } 75 \% \text { dos membros do painel de peritos encontram-se entre os scores } 1 \text { e } 3 \text {; } \\
\text { Ausência de comentários dos membros do painel de peritos que indiquem ambiguidades ou má } \\
\text { compreensão das questões. }\end{array}$ \\
\hline Sem Consenso & Todas as outras respostas. \\
\hline
\end{tabular}

a) A definição dos critérios de análise da mediana tiveram em consideração a utilização duma escala de Likert (scores 1 a 7)

Por outro lado, considerámos que seria útil caracterizar o tipo de consenso obtido em níveis de consenso (Tabela 2), relativamente aos critérios de inclusão e exclusão ${ }^{(21)}$. 
Tabela 2 - Níveis de Consenso (adaptado de FEITOSA NASCIMENTO ${ }^{(21)}$ )

\begin{tabular}{l|l|l}
\hline Consenso & Nível de consenso & Definição \\
\hline critério de inclusão & Consenso perfeito & Todos os participantes concordam completamente com a questão \\
\hline & Consenso moderado elevado & $\begin{array}{l}\text { Consenso: critérios de inclusão } \\
\text { Desvio-padrão }>1,00 \\
\text { Amplitude inter-quartil> } 1,00\end{array}$ \\
\hline critério de exclusão & $\begin{array}{l}\text { Consenso: critérios de inclusão } \\
\text { Amplitude inter-quartil superior a 1,00 }\end{array}$ \\
\hline & Consenso elevado & $\begin{array}{l}\text { Todos os participantes discordam completamente com a questão } \\
\text { Consenso: critérios de exclusão } \\
\text { Amplitude inter-quartil > 1,00 }\end{array}$ \\
\hline & Consenso moderado & $\begin{array}{l}\text { Consenso: critérios de enclusão } \\
\text { Desvio-padrão superior a 1,00 } \\
\text { Amplitude inter-quartil superior a 1,00 }\end{array}$ \\
\hline
\end{tabular}

\section{RECOLHA E ANÁLISE DOS DADOS}

O estudo Delphi desenrolou-se ao longo de três rounds, até à obtenção de consensos que permitiram a definição de um modelo de organização e partilha da informação de enfermagem entre Hospital e Centro de Saúde.

No primeiro round, tivemos como preocupação central a seleção dos membros do painel. Foi nosso propósito que os peritos fossem verdadeiros "especialistas", ou seja, que os enfermeiros tivessem um envolvimento efectivo nos processos de implementação e conhecimento nas áreas dos SIE e Classificação Internacional para a Prática de Enfermagem (ICNP/ CIPE $^{\circledR}$ ). Assim, face ao nosso conhecimento da participação de enfermeiros portugueses no desenvolvimento de projectos realizado nas áreas dos SIE e ICNP/ CIPE $^{\circledR}$, selecionamos 109 enfermeiros de diferentes áreas, verificando-se uma taxa de adesão global de $59,6 \%$, num total de 65 participantes.

Neste primeiro round, havia o interesse na obtenção das perspectivas individuais dos participantes, nomeadamente a emissão de opiniões/ideias sobre a problemática em estudo. Lançamos um primeiro questionário (Novembro/Dezembro de 2001) onde apresentamos um texto de reflexão sobre o problema em estudo, para que cada participante, após a sua leitura, desenvolvesse um "brainstorming" individual, que permitisse gerar tantas ideias quanto possível sobre o assunto em estudo. Cada perito enumerou as suas ideias de forma breve e concisa, comentando os pontos fortes ou fracos das mesmas, de acordo com a sua concepção de cuidados de enfermagem.
As respostas obtidas foram submetidas a análise de conteúdo, com o propósito geral de identificar critérios para o desenvolvimento de um modelo de organização e partilha de informação de enfermagem entre os diferentes contextos de cuidados de saúde. Definido o "corpus" de análise, constituído pelo conjunto de respostas resultantes das opiniões dos participantes, a análise teve como base a interpretação do discurso produzido, procurando unidades de informação ou temas mais comuns ${ }^{(22-23)}$, num processo de categorização (à posteriori). Neste processo procuramos identificar termos-chave que nos indicassem a significação dos conceitos que emergiam dos discursos, bem como outros indicadores que descrevessem o campo semântico desse conceito, agrupando os componentes das mensagens em "classes de sentido".

No segundo round decidimos inquirir dois grupos de enfermeiros - "peritos" e "não peritos ou afetados". Nesta etapa do estudo também pedimos a colaboração dos enfermeiros envolvidos no projeto de mudança dos SIE, que se encontrava em desenvolvimento na Unidade Local de Saúde de Matosinhos, dado que os mesmos passaram a dispor de um maior conhecimento nas áreas dos SIE e ICNP/CIPE ${ }^{\circledR}$, fruto do seu envolvimento nos processos de implementação. Neste sentido, este grupo de enfermeiros passou a integrar também o grupo de "peritos" definidos no primeiro round. Por outro lado, consideramos também pertinente recolher opiniões de enfermeiros que, embora não fossem considerados como "utilizadores bem-informados" ou "especialistas" na área dos SIE e ICNP/CIPE ${ }^{\circledR}$, necessitavam (no seu dia-a-dia) de partilhar informação de enfermagem sobre a situação dos seus utentes/doentes para assegurarem a continuidade nos cuidados. Esta estratégia constituiu uma ga- 
rantia de obtenção de pontos de vista das diferentes áreas do exercício profissional de enfermagem. Com base nestes pressupostos, decidiu-se que o grupo de enfermeiros considerados "não peritos" ou "afetados" passasse também a integrar o painel, tendo sido seleccionados globalmente 771 enfermeiros, com uma taxa de adesão global de 63,2\%, num total de 487 participantes.

O questionário relativo a este segundo round foi aplicado em Dezembro de 2002. A sua construção teve como base os resultados obtidos no round anterior, tendo englobado 45 questões. Cada participante, tendo em conta a "relevância da informação para a continuidade de cuidados de enfermagem", assinalou o score correspondente ao seu grau de concordância ou discordância com cada uma das afirmações apresentadas, podendo transmitir outras ideias ou outras questões relacionadas com o tema, que considerasse não estarem ainda contempladas.

A análise descritiva dos dados permitiu verificar que a opinião dos participantes face à globalidade do questionário apresentado foi de obtenção de consenso para a inclusão de 39 questões, com excepção para apenas 6 , que foram revistas e reescritas, sendo novamente apresentados no $3^{\circ}$ questionário deste estudo, juntamente com outras que correspondiam a novas ideias e comentários dos participantes sobre o tema em estudo.

Para o terceiro round mantivemos os critérios de selecção dos membros do painel definidos para o round anterior, procedendo à aplicação do questionário a todos os enfermeiros das mesmas unidades de saúde, independentemente de terem ou não participado no $2^{\circ}$ round. O painel foi constituído por 750 enfermeiros, com uma taxa de adesão global de $62,3 \%$, num total de 467 participantes.

O questionário que deu forma ao terceiro round foi aplicado em Setembro/2003. A sua construção teve como base os resultados do round anterior, englobando 2 partes distintas:

- Uma primeira parte, onde se apresentaram questões associadas a aspectos relativos à estrutura do modelo de partilha, e que não tinham obtido consenso para a sua inclusão ou exclusão;

- Uma segunda parte, onde se apresentaram os itens que tinham obtido consenso para a sua inclusão no modelo de partilha de informação de enfermagem e que traduziam os itens de informação a partilhar, face aos quais considerámos que seria importante definir níveis de prioridade de acesso a essa informação, em função da sua utilidade e pertinência para as diferentes situações de cuidados.

O elevado número de itens de informação (30) que, pelo seu conteúdo, foram considerados como relevantes pelos peritos no questionário anterior, levounos a recorrer a estratégias de análise e interpretação dos dados que fossem facilitadoras da especificação do respectivo modelo. Era fundamental atender à necessidade dos dados se apresentarem, no sistema de partilha de informação de enfermagem, de forma a facilitar a consulta e a tomada de decisão dos enfermeiros, o que implicaria estratégias de agregação dos mesmos.

Recorremos à análise fatorial, cuja finalidade foi associar um grande número de variáveis em grupos de variáveis, que permitissem encontrar dimensões subjacentes que identificassem constructos teóricos. O modelo de análise fatorial baseou-se na análise das componentes principais, pelo método de rotação Varimax, de acordo com os seguintes critérios:

- selecção dos itens com saturação superior a 0.30 ;

- quando um item saturasse em mais do que um factor (valor acima do referido na alínea anterior), optava-se por incluí-lo no fator em que o valor da saturação fosse mais elevado.

Seguidamente, com o intuito de definir os grandes níveis de prioridade de acesso à informação a partilhar entre os diferentes contextos de saúde, procedemos à criação de novas variáveis, resultantes da análise fatorial. A análise da estatística descritiva tornou-se fundamental na definição dos critérios de disponibilização e prioridade no acesso à informação.

Para comparar as médias dos scores entre as variáveis (perito, contexto específico de exercício profissional : unidade de cuidados, e por último, a área de exercício profissional: hospital, centro de saúde e escola de enfermagem), procedeu-se à utilização dos testes paramétricos "t-test " e "ANOVA". A comparação entre os dados permitiu verificar algumas diferenças estatisticamente significativas entre os grupos. Contudo, tal fato não teve relevância do ponto de vista da definição da estrutura dos níveis de prioridade de acesso à informação, nem dos pressupostos que suportam o modelo de organização e partilha de informação.

\section{RESULTADOS E DISCUSSÃO}

Os participantes realçaram os SIE como preponderantes para o estabelecimento de linhas de articulação entre Centro de Saúde e Hospital. Um grande número de ideias apresentadas pelos participantes permitiu caracterizar aspectos globais da partilha de informação, demonstrando uma crescente sensibilidade e consciencialização da necessidade de desenvolver SIE promotores da comunicação e continuidade dos cuidados.

Dos resultados obtidos emergiram opiniões que nos permitiram considerar um conjunto de dimensões relevantes para uma arquitectura informacional específica para a gestão dos SIE, nomeadamente: 


\section{A estrutura do modelo de partilha de informação de} enfermagem

Os participantes apontam para a importância do desenvolvimento de estruturas que sustentem a sua integração no sistema de informação global de saúde, permitindo e facilitando a troca de informação e integração com outras estruturas modulares de outros profissionais de saúde. Para os enfermeiros nem toda a informação de enfermagem é adequada para o desenvolvimento de uma estrutura automatizada de partilha de informação entre os diferentes contextos de cuidados de saúde, dado que uma grande parte dessa informação é de natureza informal, pelo que só uma parte pode ser formalizada.

Os enfermeiros salientam a necessidade da partilha de informação de enfermagem e consideram ser relevante compreender um sistema de informação de enfermagem modular, capaz de comunicar com outros módulos, o que tornará mais fácil o acesso e garantirá o encaminhamento da informação clínica acordada.

Relativamente à estrutura do modelo de partilha de informação de enfermagem foram conseguidos alguns consensos. Os enfermeiros consideram, com um nível de consenso elevado, que a partilha de informação deverá ser realizada em tempo real, com utilização da ICNP/ CIPE $^{\circledR}$ enquanto linguagem comum para a descrição dos cuidados de enfermagem. A ICNP/CIPE ${ }^{\circledR}$ assumiuse como proposta de estrutura de base e de referência para o contexto em estudo, fato ao qual não deve ser alheia a vivência experienciada pelos participantes do painel na utilização desta classificação no contexto dos SIE em uso ou nos processos formativos. Contudo, independentemente da linguagem classificada utilizada nos SIE, os participantes salientam a necessidade da existência de campos de dados, que permitam a introdução de texto livre.

A noção de que deverá ser definido um conjunto mínimo de dados de enfermagem a partilhar entre os diferentes contextos de cuidados também mereceu consenso entre os enfermeiros. Contudo, provavelmente em função da dificuldade centrada na definição de critérios, o consenso verifica-se num nível moderado, traduzido por uma maior dispersão dos dados (desvio-padrão >1,00 e um coeficiente de variação de $18,1 \%$ ).

Com um nível de consenso moderado, consideram que a informação produzida pelos enfermeiros deverá ser partilhada em articulação com a produzida pelos outros profissionais, de forma a que não apareça descontextualizada no momento da sua consulta. Quanto ao momento, a partilha de informação deverá ser realizada de forma contínua e em tempo real, mesmo que o episódio de contato de enfermagem ainda esteja a decorrer, devendo incluir dados sobre a forma de contacto com o enfermeiro do contexto hospitalar e com o enfermeiro de família (ex: nome, telefone ou e:mail,....). É evidente, que a partilha não se centrando unicamente no final dos episódios de contatos de enfermagem (ex: internamento, consulta de enfermagem) possibilitará, nomeadamente aos enfermeiros nos Centros de Saúde, o acesso à informação relativa à forma como está a decorrer o internamento, permitindo o acompanhamento da evolução dos seus utentes.

\section{Estratégias de partilha de informação}

Na utilização da informação em saúde torna-se primordial começar por definir as necessidades de informação ao nível operacional, bem como os canais de comunicação. As questões apresentadas pelos participantes englobam um conjunto de estratégias de rentabilização das potencialidades das Tecnologias da Informação e Comunicação, permitindo um mais rápido e selectivo acesso à informação.

Na perspectiva dos enfermeiros, a troca de informação entre as diversas Instituições de Saúde implica a utilização de uma Rede de Informação da Saúde, devendo ser rentabilizadas as suas potencialidades, nomeadamente na criação de canais de comunicação e interação entre os profissionais de saúde, que promovam uma atividade complementar e articulada entre os diferentes contextos de cuidados.

Face à ausência atual de uma estrutura automatizada de circulação de informação, que permita a integração das diferentes componentes de informação, não existiu consenso entre os enfermeiros relativamente à possibilidade de transcrição de dados produzidos por esses profissionais para o seu modelo de partilha da informação. Na realidade, esta estratégia com um nível de concordância "fraco", que não permite a existência de consenso, é sustentada pelo desejo de existência de uma estrutura global que permita a integração e partilha de dados de saúde, que englobem as diferentes áreas de cuidados de saúde, considerando despropositada a transcrição de informação de produção médica, remetendo-nos para a questão da redundância e da duplicação da informação. Com um nível de consenso moderado, consideram que, na ausência de uma estrutura automatizada de partilha de informação de enfermagem, a troca de informação deverá ser realizada através de relatórios em suporte de papel que sejam um output dos SIE.

\section{Protecção e segurança no tratamento dos dados}

Os dados de saúde, pelas suas características e conteúdo, são reconhecidos como constituindo um tipo de informação cujo tratamento automatizado deverá ser revestido de particulares medidas de protecção e de garantias de privacidade. Enquadradas nesta dimensão surgiram refe- 
rências centradas na proteção e segurança no tratamento dos dados. Não se verificou uma preocupação dos peritos centrada na redução dos riscos inerentes ao processo da automatização do acesso à informação, da conectividade de rede segura ou da optimização da gestão de segurança mas, uma preocupação centrada na confidencialidade dos dados e do consentimento informado.

Para os enfermeiros, a articulação entre os diferentes contextos de cuidados de saúde deverá garantir a partilha recíproca e confidencial da informação clínica relevante sobre os utentes. A partilha de informação de enfermagem relevante sobre os utentes entre os diferentes contextos de saúde deve assegurar mecanismos que levem a consentimentos informados e medidas de protecção dos dados. Com um nível de consenso elevado, os participantes apelam aos aspectos centrados na protecção de dados, remetendo para o cidadão o direito ao consentimento informado para a partilha de informação entre Hospital e Centro de Saúde.

É nesta perspectiva que se torna possível o assegurar de todo um conjunto de regras e princípios relativos aos direitos, liberdades e garantias individuais, em particular a privacidade das pessoas em causa, garantindo a sua "não discriminação" decorrente da informação partilhada. A "partilha da informação" deverá ser feita na estrita medida do necessário à prestação de cuidados de saúde, ou seja, os dados só deverão ser partilhados quando se revelem necessários à prestação de cuidados de saúde ao utente/doente ${ }^{(24)}$.

\section{O acesso à informação de enfermagem}

Os participantes do painel realçam que as necessidades de acesso à informação devem ser definidas pelos enfermeiros, tendo em consideração os seus próprios contextos de cuidados de saúde. A análise do acesso à informação centra-se na disponibilização da informação em função dos seguintes critérios: "conteúdo da informação", "período de tempo a que se reporta a informação" e "perfil do utilizador".

Com um nível de consenso elevado, consideram que o acesso à partilha de informação deverá ser determinado em função da necessidade de conteúdos específicos de informação. Este consenso é traduzido por uma baixa dispersão dos dados (desvio-padrão $<1,00$ e um coeficiente de variação de $14,8 \%$ ).

Quanto à disponibilização da informação em função de um critério temporal ou do perfil do utilizador, os enfermeiros apresentam um nível de consenso moderado. Relativamente a esta última questão, consideram que não deverá existir diferenciação entre os enfermeiros quanto ao perfil de acesso à informação, já que todos eles necessitam da informação relevante para promover a continuidade nos cuidados. Os enfermeiros apresen- tam consensos diferentes relativamente aos níveis de prioridade definidos, apresentando um nível de consenso elevado associado à existência de um segundo nível de acesso à informação, que permita a visualização de todo o histórico de dados da informação de enfermagem documentada. Ou seja, é consensual a existência dum primeiro nível de acesso que permita a visualização imediata da informação considerada relevante (definida através do conjunto mínimo de dados de enfermagem a partilhar entre os diferentes contextos de cuidados) e outros níveis de acesso que permitam a visualização à posteriori de outra informação considerada menos relevante. Desta forma, os enfermeiros poderão ter uma visualização imediata de dados relativos às situações actuais que requerem cuidados de enfermagem, e outro nível de acesso à visualização de informação complementar, nomeadamente de todo o histórico de dados da informação de enfermagem documentada.

A noção de que o modelo de partilha de informação deveria permitir a possibilidade de acesso a toda informação, mesmo aquela que não está incluída no resumo mínimo de dados a partilhar, não obteve consenso, apresentando níveis de alta dispersão das opiniões com um valor de coeficiente de variação $30 \%$ e uma amplitude inter-quartil de 2 . Neste sentido, é reforçado o consenso anteriormente estabelecido de que deverá ser definido um resumo mínimo de dados de enfermagem a partilhar entre os diferentes contextos de cuidados de saúde.

\section{Componentes de dados/informação a integrar o modelo de partilha de informação}

Relativamente à informação a partilhar entre os diferentes contextos de cuidados de saúde, os participantes enumeram um conjunto de itens que se podem agrupar por diferentes áreas centradas nos aspectos de produção/consumo de informação e no alvo em que se centra a informação (indivíduo, família, prestador de cuidados). Assim, a partilha de informação relativa ao indivíduo deve, na perspectiva dos enfermeiros, englobar três grandes áreas: informação de produção médica, informação de produção de enfermagem e informação organizacional.

a. componente de dados relacionada com a informação produzida pelos médicos:

Quando questionados sobre itens de informação de produção médica que deverão ser incluídos no modelo de partilha de informação de enfermagem, os enfermeiros apresentam níveis de concordância que geraram um nível de consenso moderado para todos os itens, com exceção para os "antecedentes pessoais e familiares, relacionados unicamente com a situação de doença", que não atingiu os critérios de concordância /discordância para a obtenção de consenso. 
A estrutura de partilha de informação deve, na perspectiva dos participantes, evidenciar um conjunto de itens de informação de produção médica relacionados com:

- História da doença actual

- Diagnóstico médico

- Motivo de internamento

- Exames complementares de diagnóstico

- Complicações verificadas durante o internamento

- Técnicas cirúrgicas realizadas

- Medicação prescrita

- Atitudes terapêuticas prescritas

- Orientações dietéticas transmitidas

- Imunizações realizadas

b. componente de dados relacionada com a informação produzida pelos enfermeiros:

Na opinião dos participantes, a partilha de informação de enfermagem deverá compreender todos os componentes do processo de cuidados: apreciação inicial, fenómenos/diagnósticos de enfermagem, intervenções de enfermagem e avaliação (quer da evolução da situação quer do processo em si).

Nesta dimensão surgem referências à necessidade da estrutura de partilha SIE oferecer uma organização sistematizada de informação que suporte a tomada de decisão e o juízo diagnóstico. A estrutura de partilha de informação deve, na perspectiva dos participantes, evidenciar um conjunto de itens de informação de produção de enfermagem relacionados com:

$\rightarrow$ Apreciação inicial

$\rightarrow$ Fenómenos/Diagnósticos de enfermagem

$\rightarrow$ Status dos fenómenos

$\rightarrow$ Intervenções de enfermagem

$\rightarrow$ Dados de vigilância

- componente de dados relacionada com a concepção de cuidados de enfermagem ao indivíduo:

Quando questionados sobre itens de informação de produção de enfermagem, associados à concepção de cuidados de enfermagem ao indivíduo, os enfermeiros consideraram que todos os itens deveriam ser incluídos no modelo de partilha de informação de enfermagem. Os enfermeiros apresentam níveis de concordância, que geram um nível de consenso elevado para as questões referentes aos diagnósticos de enfermagem, sua evolução e intervenções de enfermagem. Para as outras questões, o nível de consenso obtido foi moderado, tendo-se verificado um maior nível de dispersão dos dados relativamente à questão "Registos resultantes das prescrições médicas (medicação administrada/não administrada, atitudes terapêuticas realizadas, não realizadas, justificações,...)". Os comentários apresentados pelos membros do painel relativamente a esta questão, salientam que a relevância deste item se centra no caráter prescritivo da informação, já contemplado nos itens de produção de informação médica, levaram-nos a excluí-la para o $3^{\circ}$ round, mantendo-a nos itens de informação de produção médica, que pela natureza dos dados produzidos já se encontravam integrados noutro contexto de informação.

- componente de dados relacionada com a concepção de cuidados de enfermagem à família:

$\mathrm{Na}$ opinião dos participantes, a continuidade nos cuidados também deve ser perspectivada na lógica da relação estreita entre o enfermeiro e a família. A família entendida como unidade social, onde os processos familiares, com interações positivas ou negativas se vão desenvolvendo, reporta a uma componente fundamental para o processo de cuidados. A apreciação de cada família deverá constituir um conjunto de dados/informações que caracterizem a família, nomeadamente os seus recursos, tendo em vista o processo de cuidados.

À semelhança do verificado nos itens relacionados com os cuidados de enfermagem ao indivíduo, para as situações relacionadas com a família, os enfermeiros também consideraram que todos os itens deveriam ser incluídos no modelo de partilha de informação de enfermagem. Os enfermeiros apresentavam níveis de concordância, que geraram um nível de consenso elevado para as questões referentes aos diagnósticos de enfermagem e intervenções de enfermagem. Para as outras questões, o nível de consenso obtido foi moderado, tendo-se verificado maior dispersão dos dados.

\section{- componente de dados relacionada com a con- cepção de cuidados de enfermagem ao prestador de cuidados:}

Para os peritos, a continuidade nos cuidados também deve ser perspectivada na lógica da relação estreita entre o enfermeiro e o "prestador de cuidados", fundamentalmente em relação aos membros dependentes da família. Desta forma, consideraram como relevante englobar informação de produção de enfermagem, introduzindo aspectos globais de caracterização do prestador de cuidados, bem como, à semelhança das situações anteriores, todas os componentes do processo de cuidados , evidenciando um conjunto de itens de informação de produção de enfermagem relacionados como fenômenos/diagnósticos e intervenções de enfermagem.

À semelhança do verificado nos itens relacionados com os cuidados de enfermagem ao indivíduo, para as situações relacionadas com o prestador de cuidados, também consideram que todos os itens devem ser incluídos no modelo de partilha de informação de enfermagem. Os enfermeiros apresentam níveis de concordância que geram um nível de consenso moderado, para todas as questões. 
- componente de dados relacionada com a informação organizacional:

Para os participantes, a informação de carácter organizativo, logístico e administrativo também tem a sua relevância na perspectiva da continuidade de cuidados, realçando, essencialmente, áreas específicas que se prendem com a existência de estruturas de apoio (recursos da comunidade), de marcação de consultas (médicas e de enfermagem) e de identificação das instituições/ profissionais envolvidos no processo de cuidados.

Com um nível de consenso elevado, consideram que o acesso à partilha de informação deverá englobar itens relacionados com informação organizacional, nomeadamente aspectos associados aos suportes de apoio (humano, financeiro, material) e às consultas que se encontrarem agendadas. Estas questões apresentam valores de elevada concordância, com baixa dispersão dos dados.

\section{NÍVEIS DE PRIORIDADE DE ACESSO À INFORMAÇÃO}

Definidas as necessidades de acesso à informação, tornava-se fundamental a definição de diferentes níveis de acesso, que permitissem a organização do elevado número de itens de informação definidos como relevantes para a continuidade de cuidados de enfermagem. Reforçando a ideia de que a prioridade de acesso à informação a partilhar depende de vários fatores, nomeadamente da sua relevância para a tomada de decisão num determinado contexto, recorremos a uma estratégia de agregação dos diferentes itens de informação através da análise fatorial. Este procedimento estatístico permitiu extrair 10 fatores, cuja interpretação demonstrou ser relevante neste contexto, que explicam $75,90 \%$ da variância total desta dimensão do questionário. Todas as questões saturaram acima de 0,30 .

O fator 1, que denominámos de "Histórico de Fenómenos / Diagnóstico / Intervenções de Enfermagem" associa questões que refletem itens de informação relacionados com os históricos de fenômenos, diagnósticos e intervenções de enfermagem, que englobam o processo global de concepção e execução de cuidados de enfermagem. Este fator inclui 6 questões e explica $34,40 \%$ da variância total.

$\mathrm{O}$ fator 2, que denominamos de "Processo de diagnóstico/tratamento médico" associa questões que refletem itens de informação relacionados com o diagnóstico da situação de doença actual (exames complementares, diagnóstico médico e tratamento). Este fator inclui 4 questões e explica 9,70 \% da variância total.

O fator 3, que denominamos "Sistemas de apoio/ acompanhamento" associa questões que refletem itens de informação relacionados com a indicação de suportes de apoio ao indivíduo / família e com as consultas agendadas. Este fator inclui 2 questões e explica 5,80\% da variância total.

$\mathrm{O}$ fator 4, que denominamos "Parcerias de cuidados" associa questões que refletem itens de informação relacionados com a concepção e execução de cuidados de enfermagem dirigida à pessoa que desempenha o " $\mathrm{Pa}$ pel de prestador de cuidados", a informação e preparação que foi ou está a ser desenvolvida junto do prestador de cuidados. Este fator inclui 3 questões e explica 5,20 \% da variância total.

O fator 5, que denominamos "Tratamento médico e intercorrências" associa questões que refletem itens de informação relacionados com o tratamento da doença (nomeadamente prescrições terapêuticas) e complicações verificadas durante os episódios de internamento. Este fator inclui 5 questões e explica $5,10 \%$ da variância total.

O fator 6, que denominamos "Processo de concepção de cuidados de enfermagem" associa questões que refletem itens de informação relacionados com o diagnóstico e planejamento de intervenções de enfermagem, que engloba o processo de concepção de cuidados face às situações activas. Este fator inclui 2 questões e explica $3,8 \%$ da variância total.

$\mathrm{O}$ fator 7, que denominamos " $O$ prestador de cuidados" associa questões que refletem itens de informação relacionados com a identificação de quem exerce esse papel e dos seus conhecimentos/capacidades para desenvolver o papel de prestador de cuidados. Este fator inclui 2 questões e explica 3,50\% da variância total.

$\mathrm{O}$ fator 8, que denominamos "Dados adicionais e orientações para o processo de cuidados" associa questões que refletem itens de informação relacionados com dados de monitorização e vigilância, procedimentos que concretizam intervenções de enfermagem e notas gerais de enfermagem. Este fator inclui 3 questões e explica 3,10\% da variância total.

O fator 9, que denominamos "Áreas de atenção de enfermagem" associa questões que refletem itens de informação relacionados com a avaliação inicial de enfermagem e a identificação das situações que requerem cuidados de enfermagem. Este fator inclui 2 questões e explica $2,80 \%$ da variância total.

O fator 10, que denominamos "Doença actual" integra unicamente o item de informação relacionado com a história da doença actual. Este fator inclui 1 questão e explica $2,50 \%$ da variância total.

No sentido de definir com maior objetividade o nível de prioridade de acesso da informação a partilhar entre os diferentes contextos de saúde, procedemos à criação de novas variáveis, através do agrupamento das respostas aos itens que constituíam cada um dos fatores resultantes da análise fatorial, no sentido de obter para cada 
participante um valor médio correspondente à nova variável. Desta forma, a análise descritiva das novas variáveis permitiu uma nova visão dos dados. Num primeiro momento, procedeu-se à ordenação dos fatores em função das médias obtidas, que traduziu o nível de prioridade de acesso definido pelos participantes para cada uma das dimensões de informação definidas em cada fator identificado. Seguidamente, definimos os grandes níveis de acesso à informação. Para isso, recorremos à análise da estatística descritiva (Tabela 3), para nos ajudar a definir uma lógica nos critérios de disponibilização e prioridade no acesso à informação.

Tabela 3 - A organização da informação em níveis de prioridade de acesso

\begin{tabular}{|c|c|c|c|c|c|c|}
\hline $\begin{array}{l}\text { NÍVEL } \\
\text { ACESSO }\end{array}$ & FACTOR & FACTORES & Média & $\begin{array}{l}\text { Desvio } \\
\text { Padrão }\end{array}$ & Moda & Mediana \\
\hline \multirow{6}{*}{1} & 9 & Áreas de atençãode enfermagem & 1,72 & 1,03 & 1 & 1,5 \\
\hline & 10 & Doença actual & 2,29 & 1,54 & 1 & 2 \\
\hline & 6 & $\begin{array}{l}\text { Processo de concepção de cuidados de } \\
\text { enfermagem }\end{array}$ & 2,34 & 1,63 & 1 & 2 \\
\hline & 4 & Parcerias de cuidados & 2,41 & 1,17 & 2 & 2 \\
\hline & 7 & O prestador de cuidados & 2,44 & 1,64 & 1 & 2 \\
\hline & 3 & Sistemas de apoio/Acompanhamento & 2,75 & 1,6 & 1,5 & 2,25 \\
\hline \multirow{3}{*}{2} & 2 & Processo de diagnóstico/tratamento médico & 3,26 & 1,522 & - & 3 \\
\hline & 8 & $\begin{array}{l}\text { Dados adicionais e orientações para o } \\
\text { processo de cuidados }\end{array}$ & 3,56 & 2,38 & 1,33 & 3 \\
\hline & 5 & Tratamento médico e intercorrências & 3,69 & 2,1 & 1,8 & 3 \\
\hline 3 & 1 & $\begin{array}{l}\text { Histórico de Fenômenos/Diagnósticos e } \\
\text { Intervenções de Enfermagem }\end{array}$ & 3,89 & 1,87 & 2,33 & 3,5 \\
\hline
\end{tabular}

A tentativa de articular os modelos formais com a prática de enfermagem no desenvolvimento de SI, tendo em consideração aspectos associados à concepção dos cuidados de enfermagem no contexto de continuidade nos cuidados, resultou na definição de componentes de informação que agregam as dimensões resultantes da análise factorial, ordenadas e agrupadas em três grandes níveis de acesso à partilha de informação de enfermagem (Figura 1).

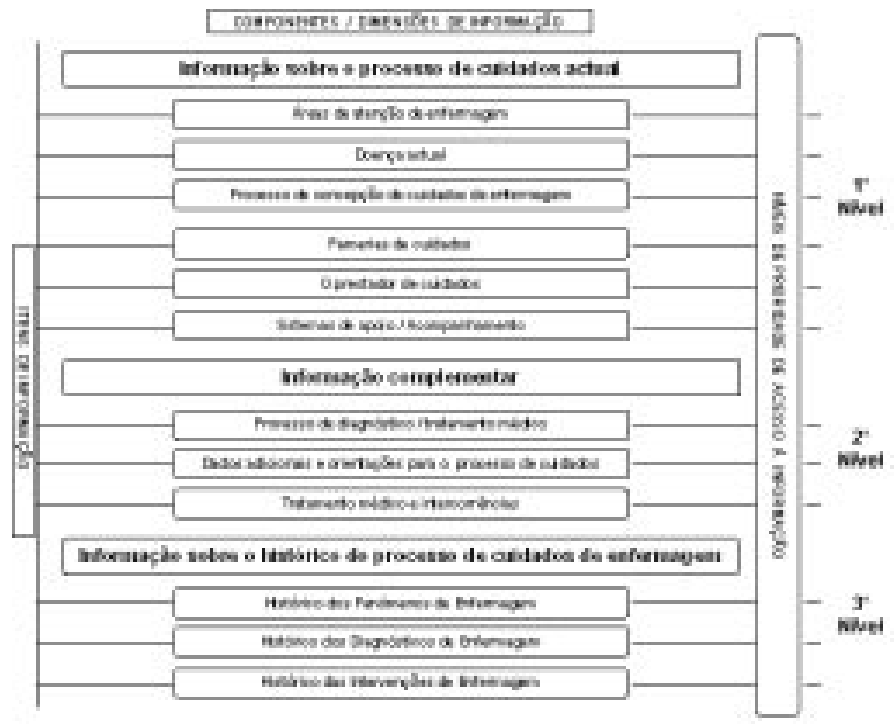

Figura 1 - Estrutura de partilha de informação entre os diferentes contextos de saúde 
A figura 1 representa os seguintes níveis de prioridade de acesso à informação:

$\rightarrow 1^{\circ}$ Nível de acesso - Informação sobre o processo de cuidados actual;

$\rightarrow 2^{\circ}$ Nível de acesso - Informação complementar;

$\rightarrow 3^{\circ}$ Nível de acesso - Informação sobre histórico do processo de cuidados de enfermagem

Da realização deste estudo Delphi emergiram consensos entre os enfermeiros, fundamentais para a definição do modelo de organização e partilha de informação de enfermagem entre hospital e centro de saúde, relativamente:

- Aos pressupostos que devem guiar o desenvolvimento do SPIE:

$\rightarrow$ Os utentes deverão dar consentimento informado para a partilha de informação;

$\rightarrow$ O modelo de partilha de informação deverá suportar-se numa estrutura que permita a sua utilização em formato de papel ou formato eletrônico;

$\rightarrow \mathrm{Na}$ ausência de uma estrutura automatizada de partilha de informação de enfermagem, o modelo deverá ter uma estrutura que permita a troca de informação através de relatórios em suporte de papel;

$\rightarrow$ A estrutura de dados do modelo de partilha de informação deverá incluir dados sobre a forma de contacto com o enfermeiro em contexto hospitalar e o enfermeiro de família;

$\rightarrow$ A informação de enfermagem deverá circular separadamente mas em articulação com a produzida por outros profissionais de saúde, de maneira a que não surja descontextualizada e permita a continuidade de cuidados;

$\rightarrow$ A partilha de informação deverá ser realizada em tempo real;

$\rightarrow$ A partilha de informação de enfermagem deverá ser contínua e em tempo real, mesmo que o episódio de contacto com o doente ainda esteja a decorrer (internamento ou consulta de enfermagem);

$\rightarrow$ Na documentação do processo de concepção de cuidados de enfermagem, os enfermeiros partilham preferencialmente os termos definidos na (CIPE/ICNPâ);

$\rightarrow \mathrm{Na}$ partilha de informação clínica de enfermagem relevante entre os diferentes contextos de saúde, o SIE deverá permitir a obtenção automática de um resumo mínimo de dados de enfermagem do SIE, que facilite a continuidade de cuidados em qualquer contexto de cuidados de saúde;

$\rightarrow \mathrm{O}$ acesso à informação de enfermagem deve obedecer às regras definidas para os utilizadores do SIE em utilização em cada Unidade de Cuidados de Saúde:
Todos os enfermeiros deverão ter o mesmo perfil de acesso à informação;

$\rightarrow$ O modelo de partilha de informação deverá permitir que o enfermeiro determine o acesso à informação pretendida através de um critério temporal (visualização de informação relativa a um determinado período de tempo);

$\rightarrow$ O modelo de partilha de informação deverá permitir que o enfermeiro determine o acesso à informação pretendida, tendo em consideração o conteúdo da informação (apreciação inicial, diagnósticos de enfermagem, intervenções de enfermagem);

$\rightarrow \mathrm{O}$ modelo de partilha de informação deverá ter diferentes níveis de acesso à informação: um primeiro nível de acesso que permita a visualização imediata da informação considerada relevante (definida através do resumo mínimo de dados de enfermagem a partilhar entre os diferentes contextos de cuidados) e outros níveis de acesso que permitam a visualização à posteriori de outra informação considerada menos relevante.

- À informação processada pelos enfermeiros nos diferentes contextos de cuidados de saúde que deverá ser efetivamente partilhada entre hospital e centro de saúde:

$\rightarrow 1^{\circ}$ NÍVEL - INFORMAÇÃO SOBRE O PROCESSO DE CUIDADOS ACTUAL

$\rightarrow$ Áreas de atenção de enfermagem

- Apreciação inicial

- Fenômenos de Enfermagem ativos

$\rightarrow$ Doença actual

- História da doença actual

$\rightarrow$ Processo de concepção de cuidados de enfermagem

- Diagnóstico de Enfermagem ativo

- Intervenções de enfermagem planeadas

$\rightarrow$ Parcerias de cuidados / O prestador de cuidados $^{1}$

- Identificação do prestador de cuidados

- Papel do prestador de cuidados

- Conhecimentos e capacidades do prestador de cuidados

- Intervenções de enfermagem dirigidas ao prestador de cuidados

- Histórico das intervenções de enfermagem dirigidas ao prestador de cuidados

$\rightarrow$ Sistemas de apoio / Acompanhamento

- Suportes de apoio

- Consultas agendadas 
$\rightarrow 2^{\circ} \quad$ NÍVEL $\quad-\quad$ INFORMAÇÃO COMPLEMENTAR

$\rightarrow$ Processo de diagnóstico / Tratamento médico e intercorrências ${ }^{2}$

- Diagnóstico médico

- Motivo de internamento

- Exames complementares de diagnóstico

- Complicações verificadas durante o internamento

- Técnicas cirúrgicas realizadas

- Medicação prescrita

- Atitudes terapêuticas prescritas

- Orientações dietéticas transmitidas

- Imunizações realizadas

$\rightarrow$ Dados adicionais e orientações para o processo de cuidados

- Dados de vigilância e monitorização

- Procedimentos das intervenções de enfermagem

- Notas gerais de enfermagem

$\rightarrow 3^{\circ}$ NÍVEL - INFORMAÇÃO SOBRE O HISTÓRICO DO PROCESSO DE CUIDADOS DE ENFERMAGEM

$\rightarrow$ Histórico de Fenômenos / Diagnósticos e Intervenções de Enfermagem

- Histórico dos Fenômenos de Enfermagem

- Histórico dos Diagnósticos de Enfermagem

- Histórico das Intervenções de enfermagem

\section{CONSIDERAÇÕES FINAIS}

Este momento de evolução dos SIE, permite potencializar a utilização dos SIE desenvolvidos e implementados nos diferentes contextos de cuidados de saúde. A capacidade de agregação da informação a diferentes níveis permite a obtenção de um resumo mínimo de dados de enfermagem (definido no modelo de organização e partilha de informação de enfermagem), reforçando a ideia de que

"o fato de no modelo de SIE se utilizar a ICNP/CIPE® enquanto vocabulário controlado para o processamento de dados, bem como o fato da estrutura concebida alojar a informação processada, permitindo distinguir as entidades: diagnósticos, status e intervenções de enfermagem - permite-nos antever a sua utilidade no fornecimento de informação a resumos mínimos de dados de enfermagem que venham, no futuro, a ser estabelecidos $^{(25)}$.

O desenvolvimento deste modelo de partilha de informação e de articulação dos Sistema de Informação de Enfermagem entre o hospital e o centro de saúde, tem como objetivo fundamental, melhorar de modo significativo e através da continuidade de cuidados, $o$ acesso e a qualidade dos cuidados de enfermagem. Pretende-se apostar na informatização e inter-comunicabilidade dos SIE, viabilizando uma maior circulação e partilha de informação, com o aproveitamento das novas tecnologias para a prestação de um melhor e mais rápido serviço ao cidadão.

\section{REFERÊNCIAS}

1. Rodrigues Filho J. The complexity of developing a nursing information system: a brazilian experience. Comput Nurs. 2001;19(3):98-104.

2. Marin HF. Vocabulários em enfermagem: atualizações e as novas iniciativas mundiais. Rev Paul Enferm. 2000;19(1):34-42.

3. Marin HF. Nursing informatics in Brazil: a brazilian experience. Comput Nurs. 1998;16(6):327-32.

4. Davenport T. Ecologia da informação: porque só a tecnologia não basta para o sucesso na era da informação. São Paulo: Futura; 1998.

5. Portugal. Assembleia da República. Lei n. 48, de 24 de agosto de 1990. Lei de Bases da Saúde. Diário da República Electrónico [periódico na Internet]. Lisboa; 1990. [citado 2004 Jun 15]. Disponível em: http://www.dr.incm.pt

6. Justo C. Critérios consensuais da qualidade do desempenho dos centros de saúde-metodologia da combinação da técnica Delphi com a opinião de informadores-chave da comunidade. Rev Port Saúde Pública. 1995;13(4):31-47.

7. Dalkey NC, Brown BB, Cochran SW. Use of self-ratings to Improve Group Estimates. Technological Forecasting Social Change. 1970;1(3):283-91.

8. Linstone HA, Turoff M. The Delphi Method: techniques and applications [text on the Internet]. 2002 [cited 2002 May 10]. Available from: http://www.is.njit.edu/pubs/delphibook/

9. Wright JTC, Giovinazzo RA. Uma ferramenta de apoio ao planejamento prospectivo. Cad Pesq Adm. 2000;1(12):13-7.

10. Bordas J, Santos J, Zaragoza F. Estudio prospectivo Delphi sobre futuros escenarios del medicamento en España. FARMAINDUSTRIA [serial on the Internet] 2002. [cited 2003 Aug 18] Available from: http://www.farmaindustria.es/ farmaweb/7pb43811 prod.nsf/0/9ca4cdb2a19448 dec1256beb 0036a12b/\$FILE/DELPHI-0-3.pdf

11. Gupta U, Clarke R. Theory and applications of the Delphi Technique: a bibliography (1975-1994). Technological Forecasting Social Change. 1996;53(2):185-211.

12. Woundenberg F. An evaluation of Delphi. Technological Forecasting Social Change. 1991;40(2):131-50. 
13. Lynn MR, Layman EL, Englebardt SP. Nursing administration research priorities: a national Delphi study. J Nurs Adm. 1998;28(5):7-11.

14. Moreno-Casbas T, Martin-Arribas C, Orts-Cortes I, CometCortes P. Identification of priorities for nursing research in Spain: a Delphi study. J Adv Nurs. 2001;35(6):857-63.

15. Millan M. La necessidad de participación en el diseño de politicas medioambientais. In: 6 a Jornada de Política Económica: Mercados, Políticas e Instituciones; 2004 Jul; Barcelona. [texto na Internet]. [cited 2004 Dec 10]. Disponível em: http:// www.ub.es/graap/mario\%20solino\%20millan.pdf

16. Fransi EC. Conceptualización de la calidad de servicio al cliente percibida en el comercio electrónico: evaluación y aplicación en el establecimiento virtual [tese na Internet]. Lleida: Universidad de Lleida; 2001. [cited 2002 May 10] Disponível em: http://www.tdx.cesca.es/TESIS_UdL/AVAILABLE/TDX1001102-114437/

17. Roque RF. Estudo comparativo de metodologias de desenvolvimento de SI utilizando a técnica Delphi [tese]. Florianópolis (SC): Faculdade de Engenharia, Universidade Federal de Santa Catarina; 1998.

18. Rowe G, Wright G. Delphi: reevaluation of research and theory. Technological Forecasting Social Change. 1991; 39 (3): 235-51.

19. Goossen W. Towards strategic use of nursing information in the Netherlands. Groningen: CIP-Gegevens Koninklijke Bibliotheek Den Haag; 2000.
20. Baumann MH, Stange C, Heffner JE, Light R, Kirby TJ, Klein $\mathrm{J}$, et al. Management of spontaneous pneumothorax: an American College of Chest Physicians Delphi Consensus Statement, Chest. 2001; 119(2): 590-602.

21. Feitosa WMN, Nascimento JV. As competências específicas do profissional de Educação Física que atua na orientação de actividades físicas: um estudo Delphi. Rev Bras Ciênc Movimento [periódico na Internet]. 2003 [cited 2004 May 10];11(4):[cerca de 8 p.]. Disponível em: http://www.ucb.br/ mestradoef/rbcm/downloads/artigo19-26v.11n.4.pdf

22. Bardin L. Análise de conteúdo. Lisboa: Edições 70; 1977.

23. Vala J. Metodologias das ciências sociais. Porto: Edições Afrontamento; 1986.

24. Portugal. Comissão Nacional de Produção de Dados (CNPD). Deliberação n. 51, de 3 de julho de 2001. Direito de acesso a dados de Saúde [texto na Internet]. Lisboa; 2001 [cited 2002 May 10]. Disponível em: http://www.cnpd.pt/actos/actos.htm

25. Silva AP. Sistemas de Informação em enfermagem: uma teoria explicativa da mudança [tese]. Porto (Portugal): Instituto de Ciências Biomédicas Abel Salazar,Universidade do Porto; 2001. 